\title{
A Case of Unusual Occurrence of Nasogastric Tube Feed Coming Through the Chest Tube in a 27-Year-Old Polytrauma Patient
}

\author{
Raunaq Chakraborty, Mohd Shoaib Budoo, Sukhyanti Kerai \\ Department of Anaesthesiology and Critical Care, Maulana Azad Medical College, New Delhi, India
}

\section{Abstract}

The provision of early and adequate nutritional support is vital for the successful recovery from the trauma in any form. Nutritional support is enteral unless specified otherwise as dictated by various benefits of the former. Nasogastric tubes provide effective and common mode of enteral feed due to the ease of placement and maintenance. To our knowledge, the late presentation of diaphragmatic hernia with gastric perforation in a trauma patient leading to feed coming through chest tube is very rare of the complications.

Keywords: Chest tube, diaphragmatic hernia, gastric perforation, nasogastric tube, unusual

\section{INTRODUCTION}

Nasogastric (NG) tubes are often inserted in intensive care settings, emergency department, and hospital wards. The common indication for NG tubes is gastric decompression to palliate bowel obstruction, preventing aspiration in intubated patients and for the delivery of enteral nutrition or medication. A case of unusual occurrence of gastric tube feed coming through the intercostal chest drain in a 27 -year-old neurosurgical patient is presented here.

\section{Case Report}

A 27-year-old male was shifted in a comatose condition to our Intensive Care Unit (ICU). The patient was a driver by profession and had a road traffic accident involving a head on collision with another vehicle.

On examination, his respiratory rate was $34 / \mathrm{min}$, pulse was 120 beats/min, and blood pressure was 130/84 mmHg. He had multiple abrasions over the left side of the face. No other external injury was noted. His Glasgow Coma Score was 9/15. He was received from the emergency room (ER) of the same hospital where he was intubated with size $\# 8.00 \mathrm{~mm}$ ID endotracheal tube.

He had fracture in his left humerus which was stabilized with an orthopedic cast in ER. Computed tomography (CT) scan

\begin{tabular}{|l|l|}
\hline \multicolumn{2}{|c|}{ Access this article online } \\
\hline Quick Response Code: & Website: \\
\hline & www.ijccm.org \\
\hline
\end{tabular}

of brain showed features suggestive of diffuse axonal injury and X-ray chest showed multiple rib fractures in the left side of the chest with pneumothorax for which an intercostal drain (ICD) was put by the surgery department in the ER. Ultrasound (USG) Focused Assessment with Sonography for Trauma (FAST) done in the casualty was negative and had shown no collection of free fluid or effusion.

On receiving this intubated patient in the ICU, he was coupled to mechanical ventilator while multichannel monitor was connected. A thoracic epidural at T6-7 space was secured and a continuous infusion of local anesthetic agent was given in view of providing analgesia for the rib fractures. A triple-lumen central venous catheter was put in the right internal jugular vein. Figure 1 depicts the $\mathrm{X}$-ray of the patient on day 1 of his ICU stay.

The patient was being managed conservatively in the ICU with initiation of NG tube feeding when on day 2 his arterial blood gas (ABG) worsened and his $\mathrm{ABG}$ showed that he

Address for correspondence: Dr. Mohd Shoaib Budoo, Flat No. 3,77/2, Khatri Nivas, B Block, Paryavaran Complex, Saket, New Delhi, India. E-mail: shoaib.budoo@gmail.com

This is an open access journal, and articles are distributed under the terms of the Creative Commons Attribution-NonCommercial-ShareAlike 4.0 License, which allows others to remix, tweak, and build upon the work non-commercially, as long as appropriate credit is given and the new creations are licensed under the identical terms.

For reprints contact: reprints@medknow.com

How to cite this article: Chakraborty R, Budoo MS, Kerai S. A case of unusual occurrence of nasogastric tube feed coming through the chest tube in a 27-year-old polytrauma patient. Indian J Crit Care Med 2018;22:547-51. 
was retaining carbon dioxide. The following findings were noted:

- $\mathrm{PaO}_{2}-80 \mathrm{mmHg}$

- $\mathrm{PaCO}_{2}-55 \mathrm{mmHg}$

- $\mathrm{pH}-7.30$

- $\mathrm{HCO}_{3}-25 \mathrm{meq} / \mathrm{L}$.

Accordingly, ventilator adjustments were made and chest X-ray (CXR) was advised. Figure 2 depicts the repeated CXR.

Finally, on day 3, his ABG picture remained the same; there was slight increase in his airway pressures as shown by the ventilator and an unusual occurrence was reported by the nursing staff on duty that the feed given through the NG tube was coming out through the chest drain.

Immediately, an urgent CXR and a contrast-enhanced $\mathrm{CT}$ (CECT) scan of thorax were ordered and NG tube feeding was withheld. In the meantime, a fiberoptic bronchoscopic evaluation was done of the tracheobronchial tree for any injury which was found to be within normal limits.

His X-ray picture [Figure 3] showed complete whiteout of the left lung.

On day 3, before the CECT thorax, his ABG showed the following:

- $\mathrm{pH}-7.225$

- $\mathrm{PO}_{2}-127$

- $\mathrm{PCO}_{2}-79.3$

- $\mathrm{HCO}_{3}-31.3$

- $\mathrm{BE}-1.6$.

CECT films revealed left hydropneumothorax with ICD in situ, left hemidiaphragmatic injury with herniated stomach in the left thoracic cavity, pleural collection of contrast given orally through NG tube probably due to a defect in stomach wall, and rib fractures between 5 and 10 ribs on the left side [Figures 4-6].

As soon as diagnosis was made, an urgent surgery referal was made and the patient was shifted to the operating room for an urgent repair of the diaphragmatic and stomach defects and reduction of the gastric contents from the thoracic cavity. Intraoperatively, a large rent was noted in the left hemidiaphragm with splenic laceration measuring $5 \mathrm{~cm}$ and gastric herniation into the hemithorax. A small gastric perforation was noted along the greater curvature.

\section{Discussion}

Traumatic diaphragmatic rupture is an uncommon, difficult-to-diagnose, and potentially life-threatening injury. Nearly $0.8 \%-5 \%$ of diaphragmatic injuries develop into diaphragmatic rupture. ${ }^{[1]}$ Blunt thoraco-abdominal trauma accounts for $74 \%$ of all diaphragmatic hernias. ${ }^{[2]}$ Most traumatic diaphragmatic ruptures occur on the left side. Right-sided ruptures are rare due to the protective effect of the liver or the strength of the right dome of the diaphragm. ${ }^{[2]}$

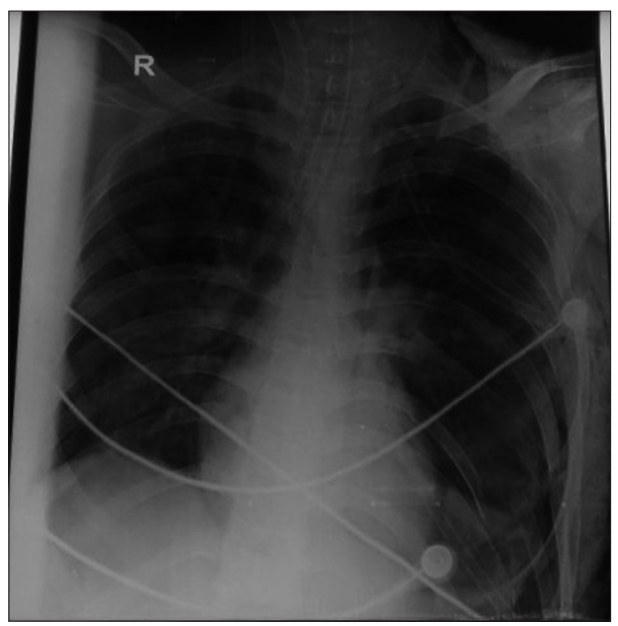

Figure 1: Chest $\mathrm{X}$-ray on day 1 in the Intensive Care Unit

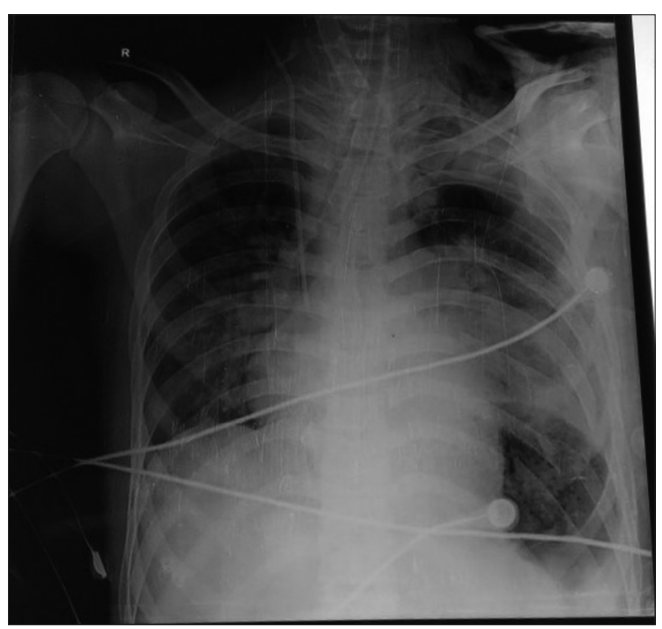

Figure 2: Chest X-ray on day 2 in the Intensive Care Unit

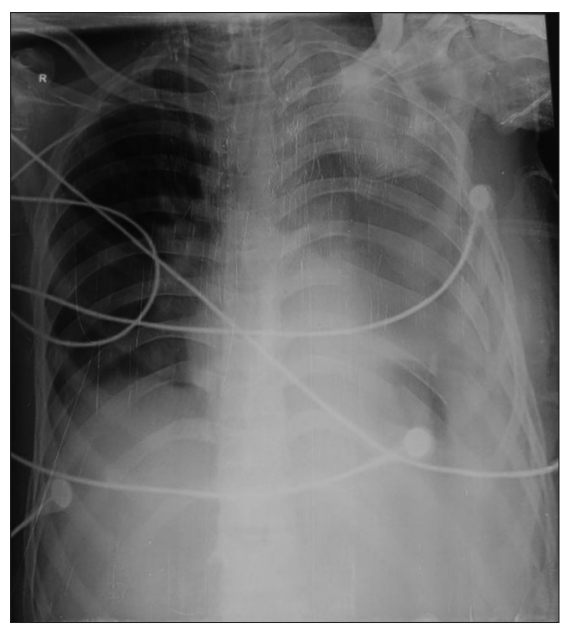

Figure 3: Chest X-ray on day 3 with left lung whiteout

Mostly traumatic diaphragmatic rupture presents with nonspecific and vague symptoms at the time of trauma either alone or in association with symptoms related to other organ injuries. The diagnosis may be missed in the acute phase in 


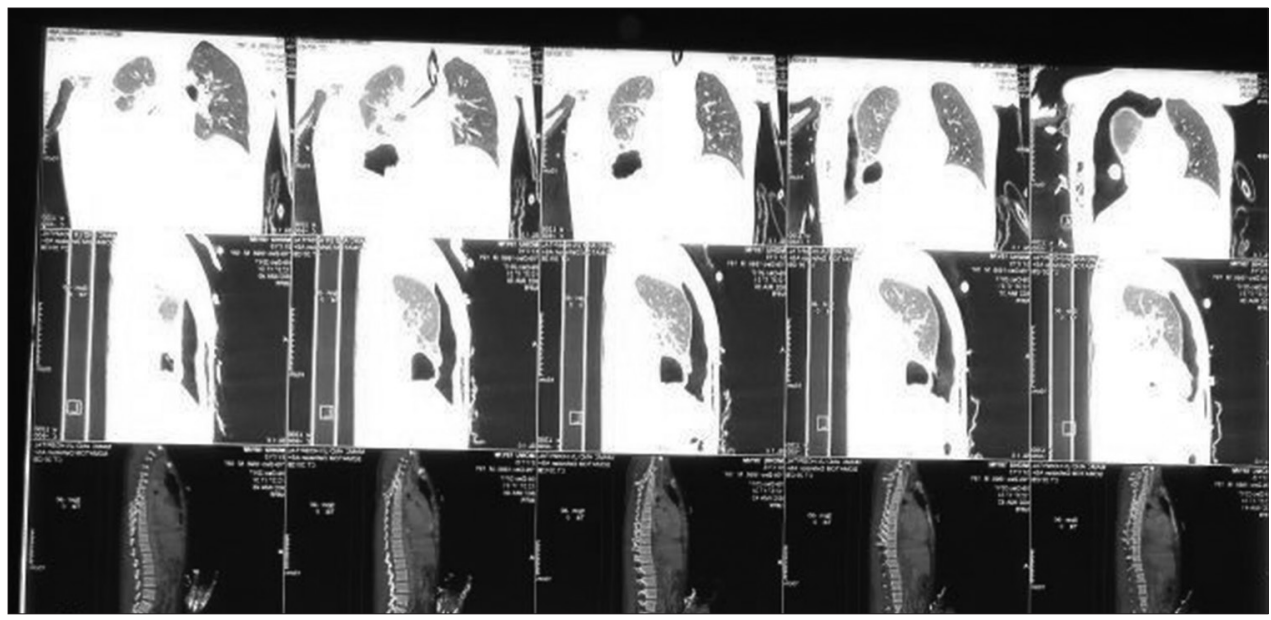

Figure 4: Contrast-enhanced computed tomography section showing left hemithorax with herniation

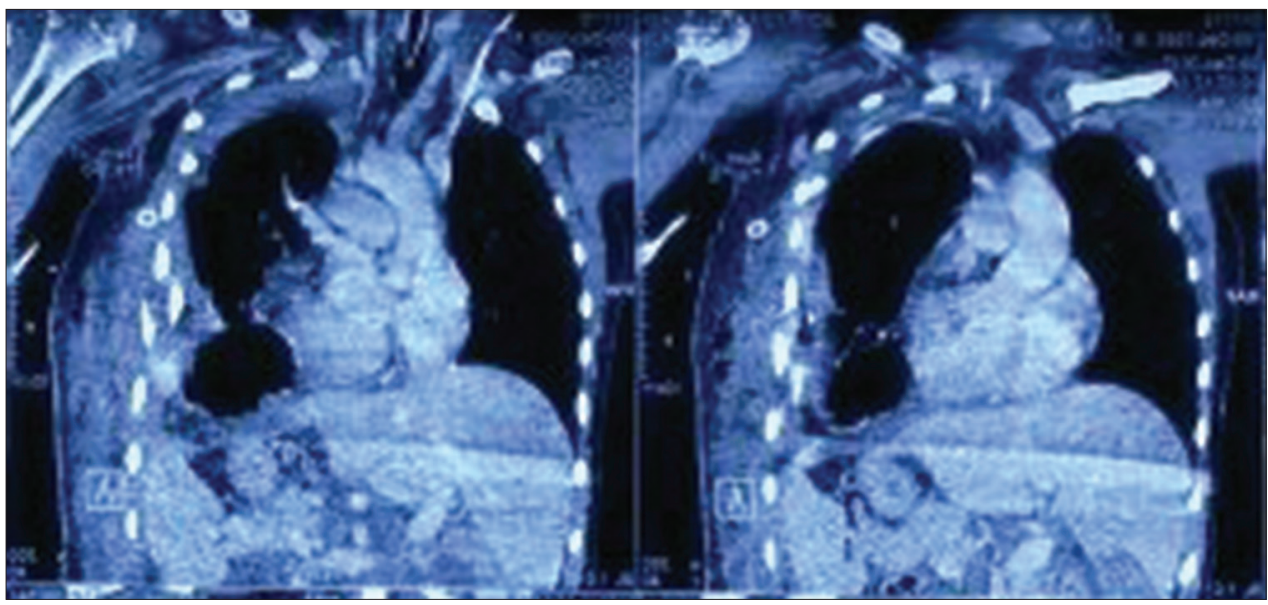

Figure 5: Contrast-enhanced computed tomography film reflecting stomach in the left hemithorax

about $30 \%$ of the cases. ${ }^{[3]}$ Sirbu et al. had observed that the ruptured diaphragmatic muscles may continue to provide a barrier across the two cavities until necrosis develops in devitalized muscles through which herniation of abdominal viscera occurs and the symptoms are produced when obstruction occurs in the incarcerated viscera. ${ }^{[4]}$

USG abdomen showed FAST positive in $33.3 \%$ of traumatic diaphragmatic hernias. ${ }^{[5]}$ In our present case, the USG FAST done in the emergency department missed the diaphragmatic injury and chest drain was put to manage the pneumohemothorax early on day 1 . Herniation was finally confirmed when symptoms appeared on day 3 . When the ICD was inserted initially, air with a small amount of blood was drained. As feeding was started on day 3, few hours later, the contents of Ryles tube feed were noticed to be coming through the chest drain.

The diagnosis is usually missed initially due to concurrent severe abdominal and thoracic visceral injuries and lack of awareness of various signs of diaphragmatic injury on imaging. A high level of suspicion is needed in these injuries. Intrathoracic injuries which are commonly associated with diaphragmatic rupture include multiple rib fractures, pneumothorax, hemothorax, and chest contusions. As in our patient, not uncommonly, diaphragmatic injury has been diagnosed as hydropneumothorax in literature in both acute and chronic settings. ${ }^{[6,7]}$ The radiological features on CXR suggestive of possibility of diaphragmatic herniation include elevated hemidiaphragm, irregular diaphragm outline, gas bubbles in chest, NG tube in chest, and compression atelectasis of lower lobe of lung. ${ }^{[8]}$

It may be hypothesized that the collected blood in the pleural cavity maintained near-normal transdiaphragmatic pressure gradient that kept the continuity of the traumatized diaphragm. Abrupt restoration of or even creation of further negative intrapleural pressure following the ICD insertion resulted in precipitation of diaphragmatic rupture and hernia. The above finding was already hypothesized by Mehrotra et al. ${ }^{[9]}$ In other literatures, it was found that Taheri and Stern have suggested that abrupt alteration in transdiaphragmatic pressure gradient can transform an asymptomatic rupture into a symptomatic hernia. ${ }^{[10]}$ This also partially explains why only some cases of diaphragmatic rupture develop hernia while others escape, as observed by Kozak et al. ${ }^{[11]}$ 


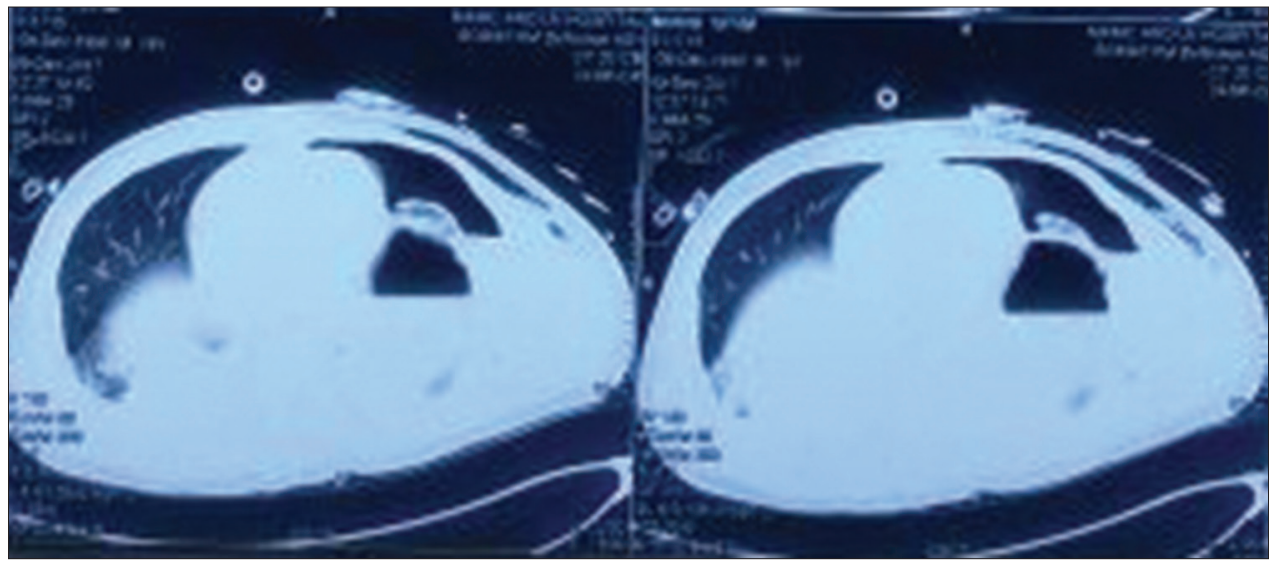

Figure 6: Section showing pleural collection of contrast given through NG tube

The timing of herniation of abdominal contents through diaphragmatic tear depends on its size. When the defect of the diaphragm is small, it may go unnoticed for a long time. In cases where the diaphragmatic defect is large as in our case, the abdominal contents herniate in immediate period after trauma.

The insertion of chest drain in view of hydropneumothorax in our patient most likely resulted in stomach perforation.

The possibility of NG insertion causing stomach perforation is unlikely as it was achieved smoothly and no force was needed during its insertion.

In suspicious cases of blunt trauma of chest and whenever possible, NG should be inserted before chest tube. The position of NG should be confirmed by CXR by noting the position of its tip, rather than by auscultation of sound of injected air through a syringe over epigastrium.

Currently, there are no accepted guidelines that help in determining which patients are at risk for major thoracic injury in blunt trauma. Approximately half of the thoracic CT scans performed in blunt trauma are negative for major thoracic injury. Payrastre et al..$^{[12]}$ described SCRAP rule to identify blunt trauma patients at very low risk for major thoracic injury with $100 \%$ sensitivity, thereby eliminating the need for chest CT. They described five variables which are as follows:

1. Oxygen saturation (abnormal is $<95 \%$ on room air or $<98 \%$ on any supplemental oxygen)

2. Chest radiograph (abnormal is any acute change that could be due to trauma)

3. Respiratory rate (abnormal is $\mathrm{RR}>25$ )

4. Chest auscultation

5. Thoracic palpation.

If any of the above variables is positive, chest $\mathrm{CT}$ is recommended.

The authors found that in major blunt trauma patients with a Glasgow coma scale $>8$, the SCRAP rule has a $100 \%$ sensitivity.

However, the study included only retrospective chart review and included low sample size; there is major need for large multicentric, prospective studies for further validation.
Similarly, Rodriguez et al. ${ }^{[13]}$ conducted three prospective multicentric trials to guide the use of CXR and CT, using two NEXUS CXR and NEXUS CT decision instruments.

They found that CXR was the best screening criterion for injury seen on chest CT and therefore advocated for the continued use of portable CXR as the most logical initial modality of chest imaging for most blunt trauma patients. If injuries are well characterized by CXR and there is no suspicion for other major injury, further imaging is unnecessary.

Therefore, currently, the decision on whether to perform a chest $\mathrm{CT}$ in blunt chest trauma patients should be made mostly by clinical judgment taking into account the clinical signs and symptoms and findings of CXR.

Currently, multidetector $\mathrm{CT}$ of chest is the modality of choice for detection of diaphragmatic injury with sensitivity and specificity of $61 \%-87 \%$ and $72 \%-100 \%$, respectively. ${ }^{[14]}$

Such a late presentation of diaphragmatic rupture leading to stomach herniation and presenting with a unique symptom of NG tube feeds coming out through the ICD has never been reported in literature to our knowledge. In our view, a high index of suspicion and early chest CT scans is the only way to clinch the diagnosis in such cases to save precious time and hence yield better results.

\section{Declaration of patient consent}

The authors certify that they have obtained all appropriate patient consent forms. In the form the patient(s) has/have given his/her/their consent for his/her/their images and other clinical information to be reported in the journal. The patients understand that their names and initials will not be published and due efforts will be made to conceal their identity, but anonymity cannot be guaranteed.

\section{Financial support and sponsorship} Nil.

\section{Conflicts of interest}

There are no conflicts of interest. 


\section{References}

1. Rossetti G, Brusciano L, Maffettone V, Napolitano V, Sciaudone G, Del Genio G, et al. Giant right post-traumatic diaphragmatic hernia: Laparoscopic repair without a mesh. Chir Ital 2005;57:243-6.

2. Kuppusamy A, Ramanathan G, Gurusamy J, Ramamoorthy B, Parasakthi K. Delayed diagnosis of traumatic diaphragmatic rupture with herniation of the liver: A case report. Ulus Travma Acil Cerrahi Derg 2012;18:175-7.

3. Pappas-Gogos G, Karfis EA, Kakadellis J, Tsimoyiannis EC. Intrathoracic cancer of the splenic flexure. Hernia 2007;11:257-9.

4. Sirbu H, Busch T, Spillner J, Schachtrupp A, Autschbach R. Late bilateral diaphragmatic rupture: Challenging diagnostic and surgical repair. Hernia 2005;9:90-2.

5. Gaine FA, Lone GN, Chowdhary MA, Lone H. The etiology, associated injuries and clinical presentation of post traumatic diaphragmatic hernia. Bull Emerg Trauma 2013;1:76-80.

6. Zieren J, Enzweiler C, Müller JM. Tube thoracostomy complicates unrecognized diaphragmatic rupture. Thorac Cardiovasc Surg 1999;47:199-202.

7. Yahya AI, Przybylski J. Non-iatrogenic perforation of the stomach by a chest tube in a patient with traumatic diaphragmatic hernia. J R Coll Surg Edinb 1998;43:62-3.
8. Shapiro MJ, Heiberg E, Durham RM, Luchtefeld W, Mazuski JE. The unreliability of CT scans and initial chest radiographs in evaluating blunt trauma induced diaphragmatic rupture. Clin Radiol 1996;51:27-30.

9. Mehrotra AK, Feroz A, Dawar S, Kumar P, Singh A, Khublani TK, et al. Diaphragmatic rupture precipitated by intercostal chest tube drainage in a patient of blunt thoraco-abdominal trauma. Lung India 2016;33:85-7.

10. Taheri MR, Stern EJ. "Delayed" right diaphragmatic rupture because of positive pressure mechanical ventilation. Curr Probl Diagn Radiol 2012;41:133-5.

11. Kozak O, Mentes O, Harlak A, Yigit T, Kilbas Z, Aslan I, et al. Late presentation of blunt right diaphragmatic rupture (hepatic hernia). Am J Emerg Med 2008;26:638.e3-5.

12. Payrastre J, Upadhye S, Worster A, Lin D, Kahnamoui K, Patterson H, et al. The SCRAP rule: The derivation and internal validation of a clinical decision rule for computed tomography of the chest in blunt thoracic trauma. CJEM 2012;14:344-53.

13. Rodriguez RM, Hendey GW, Mower WR. Selective chest imaging for blunt trauma patients: The national emergency X-ray utilization studies (NEXUS-chest algorithm). Am J Emerg Med 2017;35:164-70.

14. Kaur R, Prabhakar A, Kochhar S, Dalal U. Blunt traumatic diaphragmatic hernia: Pictorial review of CT signs. Indian J Radiol Imaging 2015;25:226-32. 